\title{
Analyzing the performance of Aloha in string multi-hop underwater acoustic sensor networks
}

\author{
Hongyang Yu, Nianmin Yao*, Shaobin Cai and Qilong Han
}

\begin{abstract}
In this article, we intend to investigate the performance of channel access protocols in multi-hop underwater acoustic sensor networks, which are characterized by long propagation delays and limited channel bandwidth. An analytical model specifically designed for contention-based protocols in multi-hop underwater acoustic networks is identified and validated. The model is based on an underwater network model, called string topology network model, which provides a method for computing the expected network throughput and the probability of packets' delivery to the gateway from an arbitrary sensor. This study demonstrates an improvement of an existing model, in which a node is implicitly assumed to be able to transmit two packets at the same time, which is not realistic due to the half-duplex character of underwater acoustic channels. Based on our findings, we propose a modified analytical model and evaluate it using NS-3 simulator. Results show that our analytical model is more precise than the existing one.
\end{abstract}

Keywords: Underwater acoustic sensor networks, Performance analysis, Medium access control (MAC) protocol, Aloha, Multi-hop

\section{Introduction}

Underwater sensor networks are becoming an important research topic. They enable a wide range of collaborative applications, such as navy military surveillance, oceanographic data collection, ocean resource exploration, disaster prevention, and so on. Since both radio and optical signals suffer significant attenuation in salty water [1], acoustic technology is the typical physical layer communication method adopted by the underwater sensor networks, namely, underwater acoustic sensor networks (UASNs). However, the speed of acoustic waves in water is only approximately $1500 \mathrm{~m} / \mathrm{s}$, which is five orders of magnitude lower than the radio propagation speed. And the bandwidth of acoustic channels in water is onethousandth that of the radio channels. Thus, compared with wireless sensor networks (WSNs), the research on UASNs is featured by long propagation delay and limited bandwidth, which pose grand challenges to almost every layer of network protocol stack and applications [2-8].

Similar to the WSNs, medium access control (MAC) protocols play a very important role in UASNs, which has

\footnotetext{
* Correspondence: yaonianmin@hrbeu.edu.cn

College of Computer Science and Technology, Harbin Engineering University, Harbin, China
}

\section{黑 Springer}

(C) 2013 Yu et al.; licensee Springer. This is an Open Access article distributed under the terms of the Creative Commons Attribution License (http://creativecommons.org/licenses/by/2.0), which permits unrestricted use, distribution, and reproduction in any medium, provided the original work is properly cited. high impact on the performance of the networks. Currently, theoretical analyses regarding MAC methods for UASNs focus on single-hop topology, which are summarized in surveys [5,9]. In particular, Xie and Cui [6] presented some theoretical analyses of contention-based protocols such as Aloha for UASNs. However, in reality, multi-hop network is more practical and it can provide wider coverage [10]. Therefore, in this article we focus on the study of multi-hop underwater scenarios.

Pure Aloha is one of the contention-based MAC protocols, which allows nodes to transmit whenever it is needed. And Aloha has become the basis of many wireless MACs since its proposal in the 1970s [11]. Due to the long propagation delay and limited bandwidth of acoustic channels, contention-based protocols tend to be effective for multi-hop UASNs [12].

In [7], the first analytical model is proposed for the contention-based protocols in string multi-hop networks, called string topology network model (STNM). The model provides a method for computing the expected network throughput and the probability of packets' delivery to the gateway from an arbitrary sensor. As a follow-up work, the performance of $p$-persistent Aloha is analyzed in [13]. In addition, a summary of these models are provided in [14]. 
In fact, the analytical model in [7] is questionable in three aspects. First, since the node cannot transmit two packets simultaneously, there is no collision among the packets sent by the same node. However, the analytical model includes the corresponding probability, and thus the collision probability of a packet reception must be higher than the actual one. Second, the packet transmitting rate is equal to the aggregate traffic rate of the same node in the analytical model. But in fact the former should be less than the latter, since the generated packet will be dropped when the node is sending packet. Third, the analytical model is not validated by the simulations or experiments, which make it less convincing.

In this article, we propose a modified analytical model of Aloha protocol for string multi-hop UASNs. In order to validate the new analytical model, we simulate Aloha protocol in a string multi-hop network via NS-3. A comparison between STNM and our analytical model is done. The results show that the analytical results obtained from our analytical model are more accurate than the ones obtained from STNM. Besides, based on our analytical model, we derive expressions for the system throughput and average end-to-end packet delay.

The remainder of this article is organized as follows. Section Performance analysis of multi-hop Aloha gives a brief overview of the string topology network, and identifies the modified analytical model of Aloha for string multi-hop UASNs. Section Experiments validates our model via simulations and analyzes the differences between the results obtained from our analytical model and the simulation results. Finally, Section Conclusion concludes the study.

\section{Performance analysis of multi-hop Aloha}

In this section, we first briefly describe the string topology network [11]. Based on the string multi-hop topology, we then present an analytical model of Aloha protocol in string multi-hop UASNs. After that, we consider the analytical model in another way, and prove that the two models are equivalent. At the end of this section, the performance expressions for string multi-hop UASNs are derived from our model.

\section{String topology network}

Same as in [7], in our analytical model we adopt the string multi-hop topology as depicted in Figure 1. In the string topology network, each node generates packets following Poisson distribution with generating rate $\lambda$, i.e., on average, each node generates $\lambda$ packets per second. Each node immediately forwards the packet received from its upstream neighbor to its downstream neighbor. And the transmission range of each node is assumed to be only capable to reach its 1-hop neighbors and the interference range is less than the distance to any 2-hop neighbor. In this article, we only consider packet loss caused by collisions. We also assume that all packets in collisions will get lost, since the differences of signal strength between packets involved in the collision are not large enough. It is further assumed a fixed packet size and uniform transmission rate for all nodes, which result in a constant packet transmission time $T$.

We notice the fact that a generated packet cannot be transmitted when the node is busy with sending. Our analytical model considers this while it is ignored by STNM. A packet sent out by each node may not follow a Poisson distribution since a packet may not be transmitted. In simulation, the packet will be dropped when it cannot be transmitted. As a result, the packet transmit rate is less than the aggregate traffic rate for each node.

As mentioned in [7], the string topology shown in Figure 1 favors the downstream traffic over the upstream traffic in underwater acoustic channels, as the packet from the downstream will be dropped only if it collides with other packets at a node further downstream.

\section{Improved analytical model}

In order to analyze the performance of Aloha for UASNs, we need to address the success rate of packet reception at

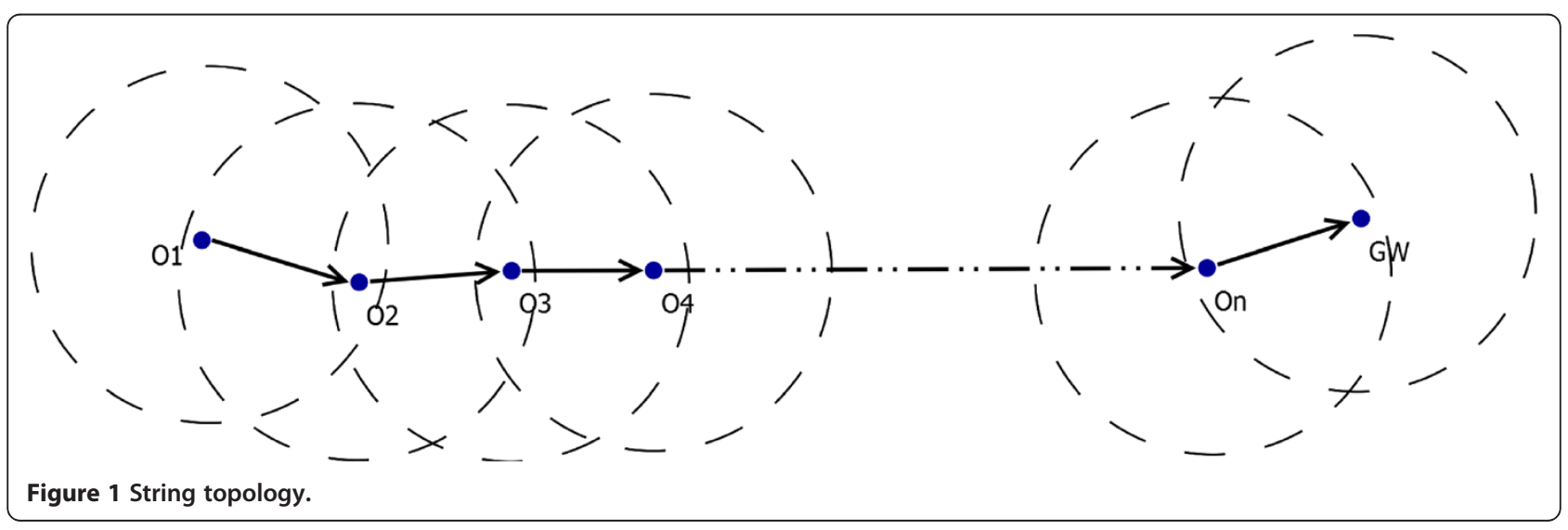


each hop. Let $\lambda_{i}$ denote the aggregate traffic rate for node $O_{i}$. The success probability of $O_{i}$ 's transmission, $P_{i}$, is the success probability that $O_{i+1}$ receives its packet, which is given as below [7]:

$$
P_{i}=\operatorname{Pr}\left\{\text { successful reception at } O_{i+1} \mid \text { packet transmitted by } O_{i}\right\}
$$

The original model (STNM) in [7] determines the likelihood that any node in the contending node set $C_{i}=$ $\left\{O_{i}, O_{i+1}, O_{i+2}\right\}$ will inject traffic such that it arrives at the reception point at any time during the reception of the packet of interest, as depicted in Figure 2. Based on the results, the model derives a series of equations relat$\operatorname{ing} P_{i}$ to $\lambda_{i}, i=1, \ldots, n$.

However, there are three disadvantages in STNM. First, the packet transmitting rate of $O_{i}$ is equal to $\lambda_{i}$ in STNM. But in fact the former is less than the latter, because some packets may not be sent out. Second, we consider the reception of a packet from $O_{i}$ at node $O_{i+1}$. The duration that $O_{i}$ takes to transmit a packet is equal to the duration $O_{i+1}$ takes to receive the packet in STNM. Due to $O_{i}$ cannot transmit two packets at the same time, there should be no collision at $O_{i+1}$ between the packets from $O_{i}$. That is, the probability of successful reception has nothing to do with whether or not $O_{i}$ is sending packets when $O_{i+1}$ is receiving a packet from $O_{i}$. Therefore, in STNM, the collision probability of a packet reception must be higher than the actual one, since the model includes the corresponding probability. At last, STNM is not validated by the simulations or experiments.

Before going further, let us denote the actual transmit rate of $O_{i}$ as $\lambda_{\text {transmit }}(i)$, and the successful transmission probability of a packet from $O_{i}$ as $P_{\text {transmit }}(i)$. The sending of a packet from $O_{i}$ can be done if and only if $O_{i}$ is not sending at that moment. Hence, the probability that a packet can be sent out by $O_{i}$ is equal to the probability that no traffic is generated by $O_{i}$ in one packet's transmission time, i.e., $T$. Recalling that the packet generation of each node follows a Poisson distribution, we have

$$
\begin{aligned}
& P_{\text {transmit }}(i)=\frac{e^{-\lambda_{i} T} \cdot\left(\lambda_{i} T\right)^{0}}{0 !}=e^{-\lambda_{i} T}, i=1, \ldots, n \\
& \lambda_{\text {transmit }}(i)=\lambda_{i} \cdot P_{\text {transmit }}(i)=\lambda_{i} e^{-\lambda_{i} T}
\end{aligned}
$$

The aggregate traffic for node $O_{i+1}$ includes the traffic received from $O_{i}$ and the one generated by $O_{i+1}$. Given that each node originates packets at the same rate, we have

$$
\begin{aligned}
& \lambda_{1}=\lambda \\
& \lambda_{i}=\lambda_{\text {transmit }}(i-1) \cdot P_{i-1}+\lambda, i=2, \ldots, n \\
& P_{\text {transmit }}(i)=e^{-\lambda_{i} T}=\left\{\begin{array}{cc}
e^{-\lambda T} & i=1 \\
e^{-\left(\lambda_{\text {transmit }}(i-1) P_{i-1}+\lambda\right) T} & i=2, \ldots, n
\end{array}\right.
\end{aligned}
$$

Furthermore, the actual transmit rate for each node is

$$
\begin{array}{rlr}
\lambda_{\text {transmit }}(i) & =\lambda_{i} \cdot P_{\text {transmit }}(i) \\
& =\left\{\begin{array}{lc}
\lambda e^{-\lambda T} & i=1 \\
\left(\lambda_{\text {transmit }}(i-1) P_{i-1}+\lambda\right) \cdot e^{-\left(\lambda_{\text {trassmit }}(i-1) \cdot P_{i-1}+\lambda\right) T} & i=2, \ldots, n
\end{array}\right.
\end{array}
$$

The successful reception of a packet from $O_{i}$ at node $O_{i+1}$ depends on the state of $O_{i+1}$. The reception of a packet will fail while $O_{i+1}$ is currently overhearing the transmission of a packet by its downstream neighbor $O_{i+2}$, or currently sending a packet to $O_{i+2}$. And the reception of a packet will succeed only if $O_{i+1}$ is idle in twice the packet's reception time, i.e., $2 T$. These constraints are independent.

Since the packets' arrival at node follows the Poisson process, the probability that no traffic is sent out by $O_{j}$ is equal to the probability that no traffic arrives at $O_{j}$.

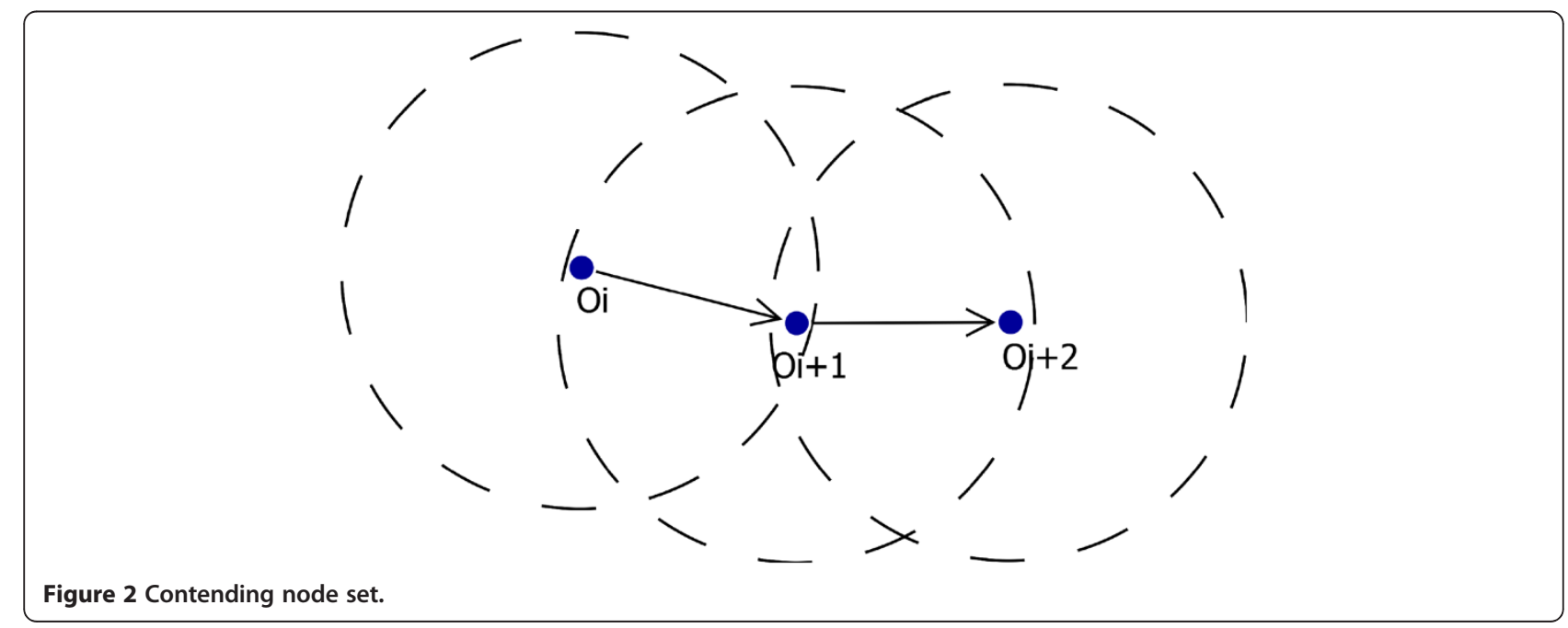


Table 1 Parameter setting

\begin{tabular}{lc}
\hline Parameter & Value \\
\hline Transmission time & $1 \mathrm{~s}$ \\
Data rate & $256 \mathrm{bps}$ \\
Packet size & $32 \mathrm{Bytes}$ \\
Total node number & 9 \\
Channel bandwidth & $10-14 \mathrm{kHz}$ \\
Transmit power & $190 \mathrm{~dB} \mathrm{re} 1 \mathrm{uPa}$ \\
Minimum SIR & $10 \mathrm{~dB}$ \\
Simulation time & $20,000 \mathrm{~s}$ \\
The position of each node & $(45,000 i, 0,100)$ \\
\hline
\end{tabular}

The probability that no packet is sent out by $O_{j}$ during a packet's reception period is

$$
\frac{e^{-\lambda_{j}(2 T)} \cdot\left(\lambda_{j}(2 T)\right)^{0}}{0 !}=e^{-\lambda_{j}(2 T)}
$$

Therefore, the probability of a successful reception of a packet from $O_{i}$ is

$$
\begin{aligned}
& P_{i}=e^{-\left(\lambda_{\text {transmit }}(i+1)+\lambda_{\text {transmit }}(i+2)\right)(2 T)}, i=1, \ldots n-2 \\
& P_{n-1}=e^{-\lambda_{\text {transmit }}(n)(2 T)} \\
& P_{n}=1
\end{aligned}
$$

Combining Equations (5) and (7), we can obtain nonlinear equations with respect to $n$ variables: $\lambda_{\text {transmit }}$
(1), $\lambda_{\text {transmit }}(2), \ldots, \lambda_{\text {transmit }}(n)$. This can be achieved by solving the following minimization problem

$$
\min _{\lambda_{\text {transmit }}(1), \lambda_{\text {transmit }}(2), \ldots, \lambda_{\text {transmit }}(n)} \sum_{i=1}^{n}\left[F_{i}^{\prime}(\Lambda)-\lambda_{\text {transmit }}(i)\right]^{2}
$$

where $\Lambda=\left(\lambda_{\text {transmit }}(1) \quad \lambda_{\text {transmit }}(2) \ldots \lambda_{\text {transmit }}(n)\right)$, and $F_{i}^{\prime}(\Lambda)$ is calculated by iterative computation using Equation (5).

The Nelder-Mead simplex method has been quite effective to solve the above minimization problems [15]. In Section Experiments, we will calculate above equations and obtain the analytical results using this method in Matlab.

\section{Equivalent modification analytical model}

Reconsidering the situation where the packet is generated when some packet is being transmitted. In this situation, the packet will be dropped, since it cannot be transmitted. We suppose this situation as a collision, though in this case there is no collision occurs actually. We term this situation as "drop collision," and we term the collision which occurs actually as "usual collision." However, there is a difference between drop collision and usual collision. In drop collision, the packet which is being transmitted will not be lost. However, all packets will be lost in usual collision. Let $P_{\text {success }}(i)$ denote the
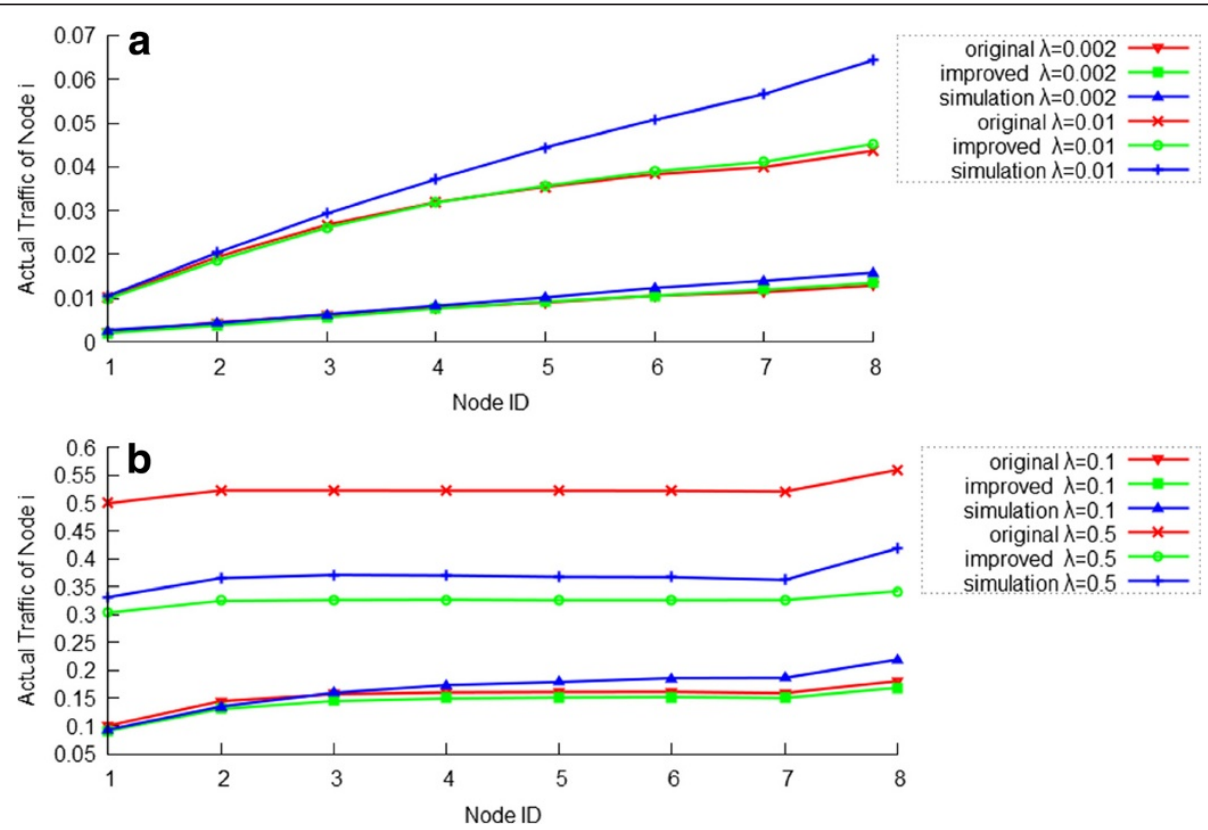

Figure 3 Original, improved, and simulation results of node actual traffic load $\left(\lambda_{\text {transmit }}(i)\right)$ versus node ID at $\lambda=0.002,0.01,0.1$, and 0.5 . 

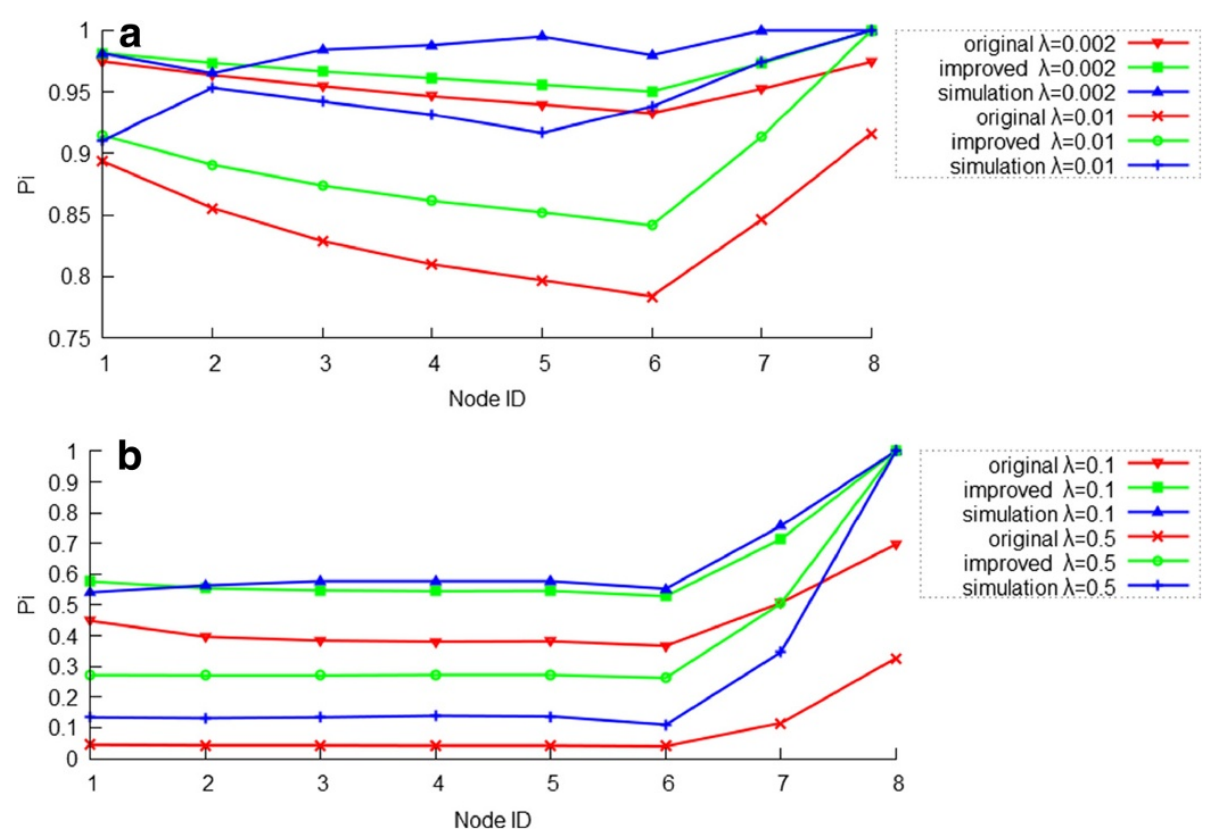

Figure 4 Original, improved and simulation results of $P_{i}$ versus node ID at $\lambda=0.002,0.01,0.1$, and 0.5 .

success probability that $O_{i+1}$ receives the packet arrived at $O_{i}$. More formally stated, that is

$P_{\text {success }}(i)=\operatorname{Pr}\left\{\right.$ successful reception at $O_{i+1} \mid$ packet arrived at $\left.O_{i}\right\}$

and others are same as above. Therefore, we have

$$
\begin{aligned}
& \lambda_{1}=\lambda \\
& \lambda_{i}=\lambda_{i-1} \cdot P_{\text {success }}(i-1)+\lambda=\lambda \cdot\left(1+\sum_{j=1}^{i-1} \prod_{k=j}^{i-1} P_{\text {success }}(k)\right), \\
& i=2, \ldots, n
\end{aligned}
$$

Following these definitions, the successful reception of a packet from $O_{i}$ at node $O_{i+1}$ depends on the state of $O_{i}$ and $O_{i+1}$. The reception of a packet from $O_{i}$ is successful only if $O_{i}$ is not sending some packet and no other packets arrive at $O_{i+1}$ during one packet's reception period. In other words, there are three possible reasons for a packet from $O_{i}$ to get lost: first, the packet generated by $O_{i}$ is tried to be sent when $O_{i}$ is transmitting some other packet; second, the packet arrives at $O_{i+1}$ when $O_{i+1}$ is currently overhearing a packet from $\mathrm{O}_{i+2}$ or $\mathrm{O}_{i+1}$ is transmitting some packet to $\mathrm{O}_{i+2}$; and third, $\mathrm{O}_{i+1}$ initiates a transmission or some packet from $\mathrm{O}_{i+2}$

arrives at $O_{i+1}$ when $O_{i+1}$ is receiving the packet. Therefore, the reception success probability at $O_{i+1}$ is

$P_{\text {success }}(i)$ $=e^{-\left(\lambda_{i+1} \cdot P_{\text {transmit }}(i+1)+\lambda_{i+2} \cdot P_{\text {transmit }}(i+2)\right) \cdot(2 T)}$
$=e^{-\left(\lambda_{\text {transmit }}(i+1)+\lambda_{\text {transmit }}(i+2)\right) \cdot(2 T)-\lambda_{i} T}$

$=P_{i} \cdot P_{\text {transmit }}(i)$

$P_{\text {success }}(n-1)=e^{-\left(\lambda_{n} \cdot P_{\text {transmit }}(n)\right) \cdot(2 T)-\lambda_{n-1} T}$

$P_{\text {success }}(n)$

$=e^{-\lambda_{\text {transmit }}(n)(2 T)-\lambda_{n-1} T}=P_{n-1} \cdot P_{\text {transmit }}(n-1)$
$=e^{-\lambda_{n} T}=1 * P_{\text {transmit }}(n)=P_{n} \cdot P_{\text {transmit }}(n)$

$, i=1, \ldots, n-2$

Combining Equations (10) and (11), we have

$$
\begin{aligned}
\lambda_{1} & =\lambda \\
& =\lambda_{i-1} \cdot P_{\text {success }}(i-1)+\lambda \\
\lambda_{i} & =\lambda_{i-1} \cdot P_{i-1} \cdot P_{\text {transmit }}(i-1)+\lambda \quad, i=2, \ldots, n \\
& =\lambda_{\text {transmit }}(i-1) \cdot P_{i-1}+\lambda
\end{aligned}
$$

We can see that this analytical model is equivalent to the one described in Section Improved analytical model.

\section{Network performance expressions}

Let $U_{i}$ denote the utilization of the link from $O_{i}$ to $O_{i+1}$. The utilization of the network and the effective 


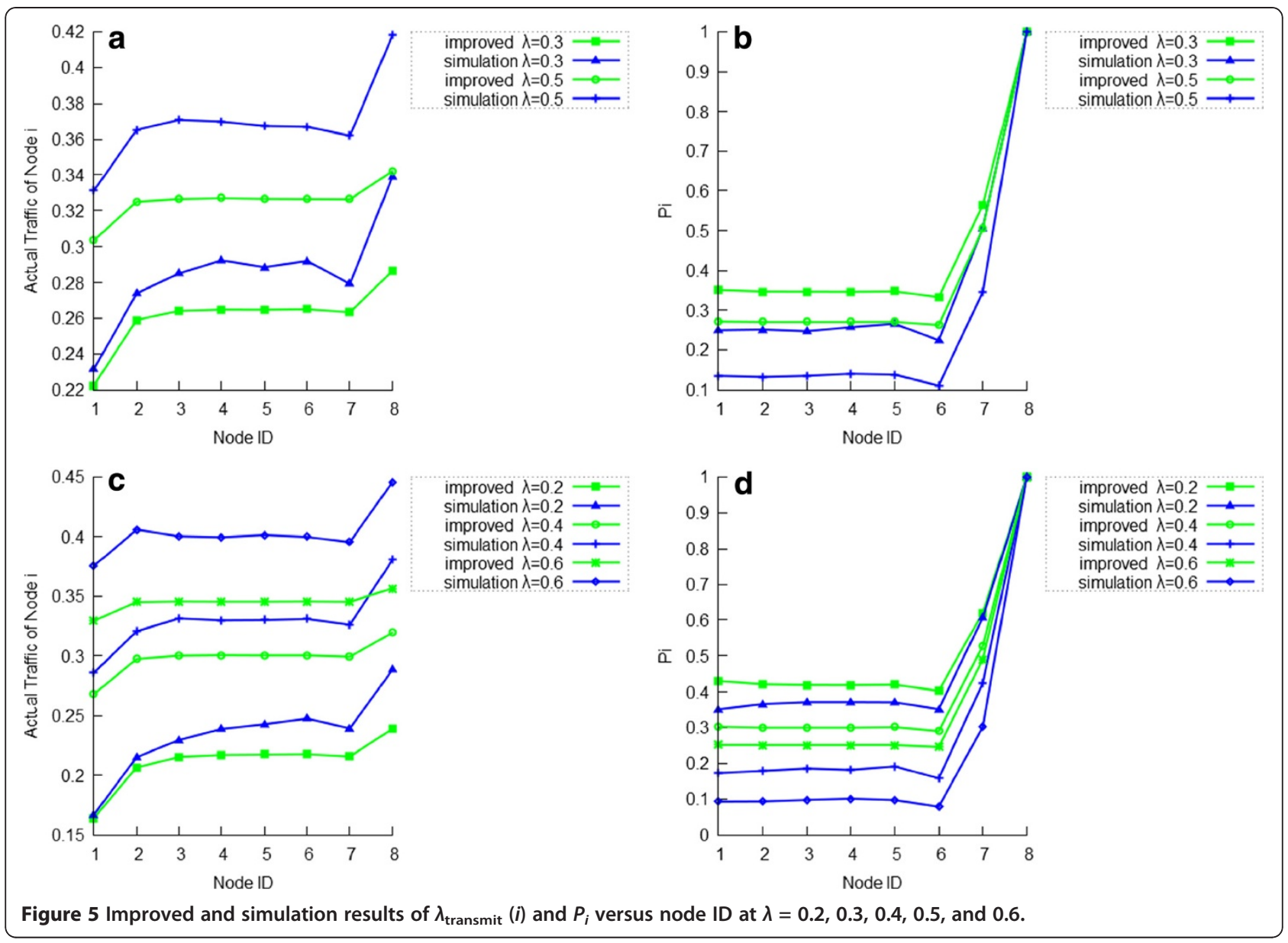

throughput of the sensor network, denoted by $U(n)$ and $S(n)$, can be expressed as follows

$$
\begin{gathered}
U(n)=U_{n}=\lambda_{\text {transmit }}(n) \cdot P_{n} \\
S(n)=\lambda_{\text {transmit }}(n) \cdot P_{n} \cdot L \cdot \alpha
\end{gathered}
$$

where $\lambda_{\text {transmit }}(n)$ and $P_{n}$ are calculated by Equations (5), (7), and (8), respectively; $L$ is the average packet size in bits; and $\alpha$ is the average fraction of data bits in each packet received by the gateway.

The end-to-end delay in networks is the sum of transmission and propagation delays at source and intermediate nodes. The average end-to-end packet delay of the network, denoted by delay, can be expressed as follows

$$
\text { de } \bar{l} \text { ay }=\sum_{i=1}^{n}\left(\left(\prod_{j=i}^{n} P_{j}\right) \cdot\left(\sum_{k=i}^{n}(T+\operatorname{Distance}(i) / c)\right)\right)
$$

where $c$ is the speed of acoustic waves in water; $T$ is the packet transmission time; and Distance $(i)$ is the distance from $O_{i}$ to $O_{i+1}$.

\section{Experiments}

In this section, we first calculate the analytical results obtained from STNM ("the original results" for short) using Equations [7] and the ones obtained from our model ("the improved results" for short) using Equations (5) and (7). Then, we perform simulation with the same parameter setting using NS-3. After that, we compare STNM with the modification model, as well as validate them with the simulation results.

The NS-3 UAN module offers accurate modeling of the underwater acoustic channel and a model of the WHOI acoustic modem. Both the attenuation and noise are known to be strong functions of frequency. In our simulation, the acoustic channel model adopts Thorp attenuation [16], which is used for the calculation of the SNR at the receiver with the consideration of the ambient noise.

Without loss of generality, $T$ is set to be $1 \mathrm{~s}$, packet size is 32 Bytes, and the simulation operation time is $20,000 \mathrm{~s}$. There are nine nodes in the string multi-hop network, so $n$ is 8 . All nodes are at the same depth, 100 $\mathrm{m}$. We use a channel bandwidth of $10-14 \mathrm{kHz}$, data rate of $256 \mathrm{bps}$, and transmit power of $190 \mathrm{~dB}$ re $1 \mathrm{uPa}$. The 


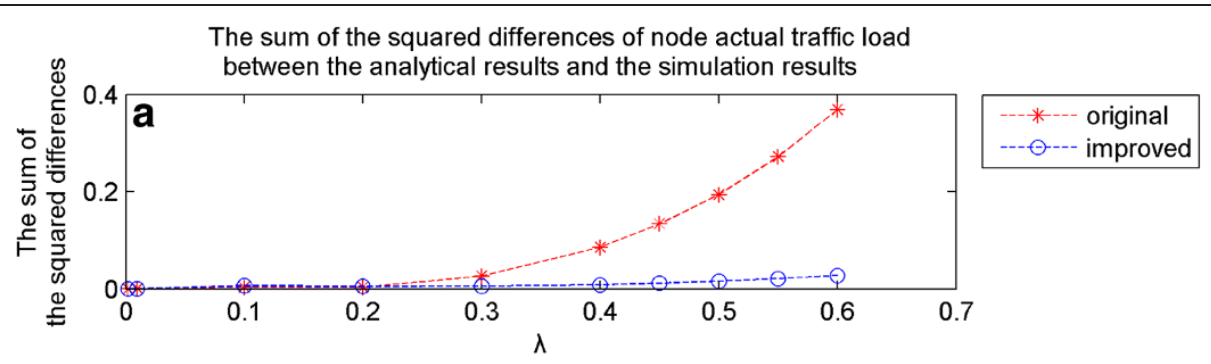

The sum of the squared differences of $\mathrm{Pi}$

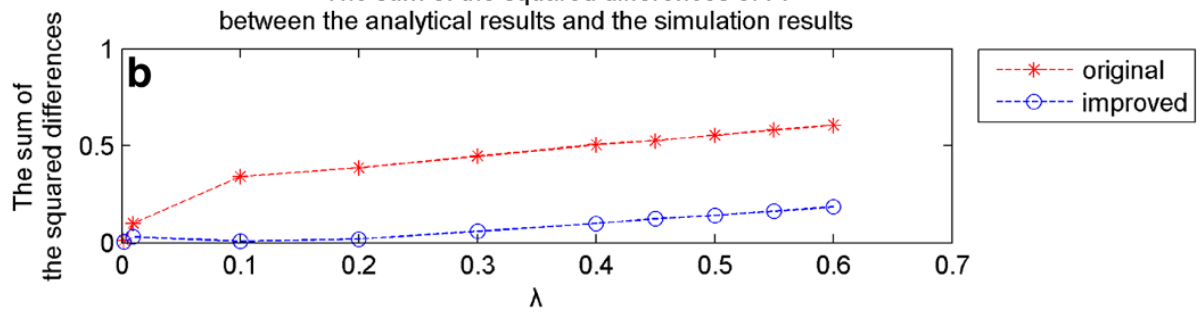

The sum of the squared differences of $\mathrm{Pi}$ except $\mathrm{Pn}$
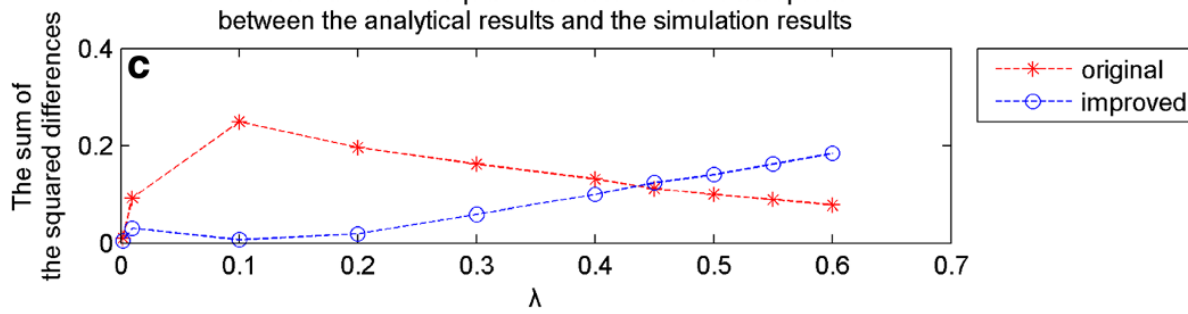

Figure 6 The sum of the squared differences between the results obtained from the analytical model and the simulation versus the $\lambda$.

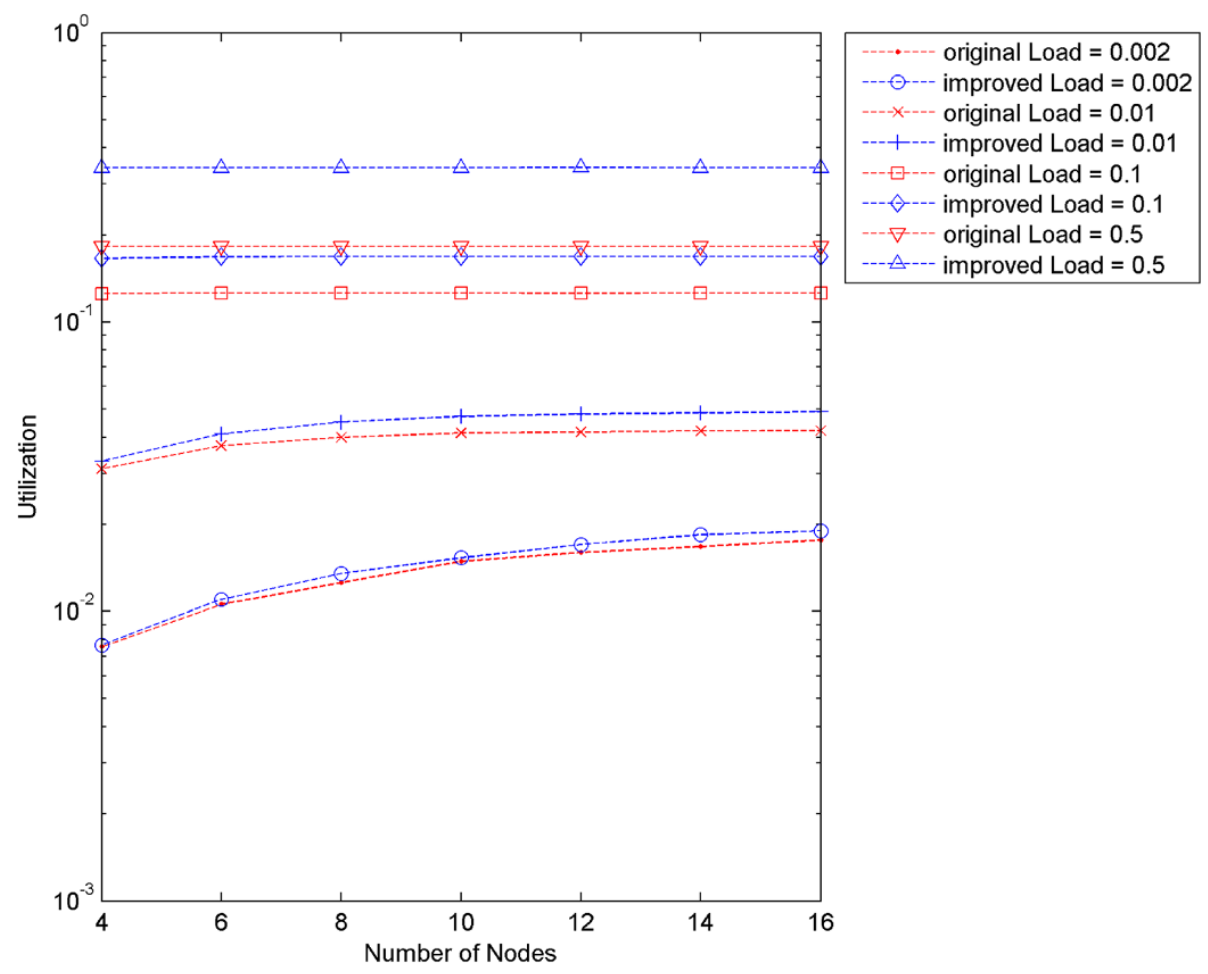

Figure 7 Original and improved results of utilization versus number of nodes at different load. 


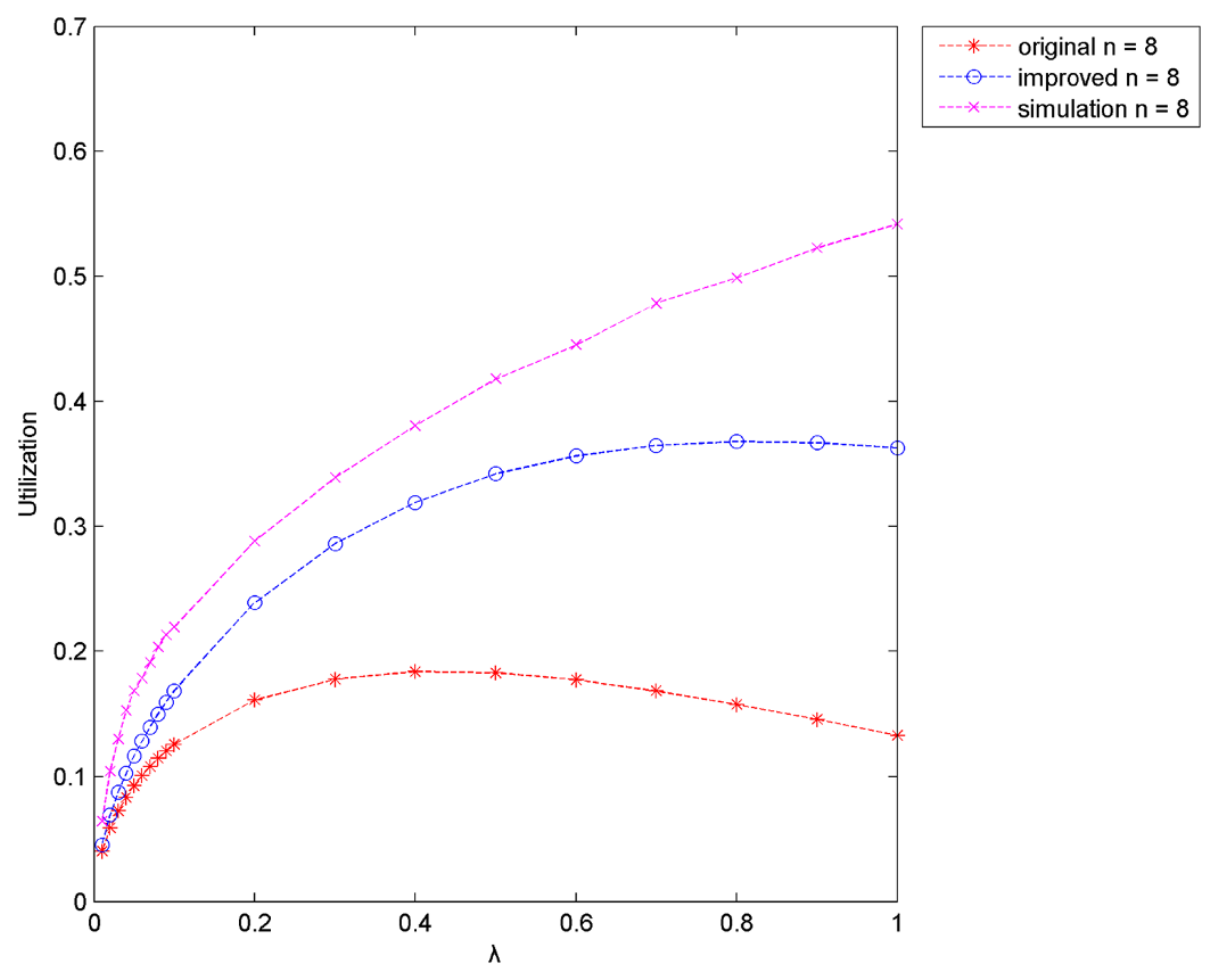

Figure 8 Original, improved, and simulation results of utilization versus $\lambda$.

minimum SIR that the receiver requires for a correct reception is set to $10 \mathrm{~dB}$. Thus, the packet transmission time is $32 \times 8 / 256=1 \mathrm{~s}$, which is equal to $T$. We use a path loss exponent of 1.5 corresponding to practical spreading, and absorption according to Thorp, sensor node's communication range is between 50509 and $50510 \mathrm{~m}$. We set the distance between nodes as $45000 \mathrm{~m}$ to make sure that a node can only talk with its one-hop neighbors. Therefore, $O_{i}^{\prime}$ s position is $(45000 i, 0,100)$ in the string topology network. All parameters are listed in Table 1.

First, $\lambda$ is set as $0.002,01,0.1$, and 0.5 , respectively, to diversify the per-sensor load like [7]. Figure 3 shows the original, improved, and simulation results of the actual traffic load of each node $\left(\lambda_{\text {transmit }}(i), i=1, \ldots, n\right)$. Same as claimed in [7], we can see that $\lambda_{\text {transmit }}(i)$ increases when $i$ increases, regardless of $n$. We can also observe that our improved results can better match the simulation results than the original ones do.

Figure 4 shows that $P_{i}$ deceases except at the last two nodes due to their smaller contending node sets. When the load exceeds $\lambda=0.5$ each node has reached its saturation status and $P_{i}$ becomes stable. In particular, no matter what load is chosen, simulation results of $P_{n}$ is always approaching to 1 , therefore our model is more accurate than STNM.
From Figures 3 and 4, we can observe that there is still a bias between our improved results and simulation results. In order to further investigate this issue, we let $\lambda=0.2$, $0.3,0.4,0.5$, and 0.6 , respectively. Figure 5 shows that the bias between our improved results and simulation results increases as the load goes up. One of the main reasons for the bias is that in our model and STNM, we assume that the packet transmitted by each node follows a Poisson process, which can approximately be satisfied when the $\lambda$ is low. However, the interarrival time between the two adjacent packets transmitted is not an exponential distribution, since it is more than $T$. Thus, with the increase of the $\lambda$, the packet transmitted by each node cannot be approximately by a Poisson process any more, which contributes to the bias in the heavy traffic-loaded network.

In the following, we consider the sum of the squared differences between the results obtained from the analytical model and the simulation. The analytical model which has smaller value is more accurate. Figure 6a shows the sum of the squared differences between the original $\lambda_{\text {transmit }}(i)$ and the $\lambda_{\text {transmit }}(i)$ measured by simulation, and the sum of the squared differences between the improved $\lambda_{\text {transmit }}(i)$ and the $\lambda_{\text {transmit }}(i)$ measured by simulation versus the $\lambda$. We can observe that our improved results can better match the simulation results than the original ones do. 
Figure $6 \mathrm{~b}$ shows the sum of the squared differences between the original $P_{i}$ and the $P_{i}$ measured by simulation, and the sum of the squared differences between the improved $P_{i}$ and the $P_{i}$ measured by simulation versus the $\lambda$. We can observe that our improved results are always more coincident with the simulation results than the original ones. Considering $P_{n}$ significantly impacts results, we compute the sum of the squared differences of $P_{i}$ except $P_{n}$, again. From Figure 6c, we can observe that the bias of our improved results still fit better than the original ones do in most cases. The above results show that our analytical model is more precise.

In the following, we explore the utilization of the string topology network. Gibson et al. [7] provide a method for computing the expected network utilization. Since STNM in [7] does not fit the simulation result well, we reconsider the utilization of the network using Equation (13).

Figure 7 shows the original and improved results of utilization versus number of nodes at different load. Figure 8 shows the original, improved, and simulation results of utilization versus load, i.e., $\lambda$. As mentioned in [7], the utilization increases with $n$ when the persensor load is small, since more packets arrive at the gateway when the nodes have not reached their saturation status. When the nodes are saturated, or almost saturated, the utilization of the network is almost flat with respect to $n$. However, Gibson et al.'s [7] analysis severely underestimated the utilization of Aloha in string topology UASNs, as depicted in Figures 7 and 8. The utilization can reach the maximum at about 0.3678 when the load is about 0.8 . The results from STNM are less than half of the results of our model. The throughput of the network is similar with the utilization.

From Figure 8, we can also observe that the utilization obtained from our model and STNM first increases with the load. After it has reached the maximum, it starts decreasing. However, the utilization obtained from simulation is monotonically non-decreasing and the maximum utilization is 1 . When the load is very heavy, only the data from the last node can reach the gateway. Thus, the bias between analytical model and simulation results increases as the load goes up. This also indicates that the packet transmitted by each node cannot be approximated by a Poisson process when $\lambda$ is high.

Although there is a bias between analytical model and simulation results, our analytical model is still reasonable, since $\lambda$ is less than 1 in most cases for underwater scenario. To further improve the analytical model's accuracy, the relationship between the bias and the load should be investigated. We would like to explore this topic in our future work.

\section{Conclusion}

In this article, we identified and validated the analytical model of Aloha in multi-hop UASNs. The simulation results justify that our modified analytical model is more accurate than STNM. Based on our model, we provide the expected network throughput and average end-toend delay in string topology underwater networks.

The above analytical models are based on pure Aloha protocol which the node's transmission has higher priority than the node's reception. It is very inefficient, since a node will simply transmit a packet whenever it has anything to send, regardless of whether it is currently receiving a packet.

In the future, we would like to investigate the relationship between the bias and the load for our analytical model as well as further improve the accuracy of our analytical model. And we would also investigate other Aloha-based protocols such as slotted-Aloha protocol and Aloha with half-duplex protocol for string topology multi-hop network.

\section{Competing interests}

The authors declare that they have no competing interests.

\section{Acknowledgment}

This study was supported by the National Natural Science Foundation of China under Grant no. 61073047; Fundamental Research Funds for the Central Universities: HEUCFT1202 and Harbin Special funds for Technological Innovation Talents: 2012RFLXG023.

Received: 25 October 2012 Accepted: 31 January 2013

Published: 12 March 2013

\section{References}

1. F Schill, UR Zimmer, J Trumpf, Visible spectrum optical communication and distance sensing for underwater applications, in Proceedings of the Australasian Conference on Robotics and Automation, 2004

2. J Liu, Z Wang, Z Peng, J Cui, S Zhou, JSL: a joint solution for localization and time synchronization underwater sensor networks, in Proceedings of the IEEE Communications Society Conference on Sensor, Mesh and Ad Hoc Communications and Networks (SECON) (Seoul, Korea). 18-21 June 2012

3. J Liu, X Han, MA Bzoor, M Zuba, J Cui, RA Ammar, S Raj, PADP: prediction assisted dynamic surface gateway placement for mobile underwater networks, in Proceedings of the Seventeenth IEEE Symposium on Computers and Communication (ISCC'12) (Cappadocia, Turkey). 1-4 July 2012

4. J Liu, Z Wang, Z Peng, M Zuba, J Cui, S Zhou, TSMU: a time synchronization scheme for mobile underwater sensor networks, in Proceedings of the GLOBECOM (Houston, TX, USA, 2011)

5. J Heidemann, W Ye, J Willis, AA Syed, Y Li, Research challenges and applications for underwater sensor networking, in Proceedings of the IEEE Wireless Communications and Networking Conference (WCNC 2006) (Las Vegas, NV), pp. 229-235. 3-6 April 2006

6. P Xie, J Cui, Exploring random access and handshaking in large scale underwater wireless acoustic sensor networks, in Proceedings of the MTS/ IEEE Oceans Conference (Boston, 2006)

7. J Gibson, G Xie, Y Xiao, H Chen, Analyzing the performance of multi-hop underwater acoustic sensor networks, in IEEE Oceans' 07 (Aberdeen, Scotland, 2007)

8. J Liu, Z Zhou, Z Peng, J Cui, Mobi-Sync: efficient time synchronization for mobile underwater sensor networks, Proceedings of the GLOBECOM (Miami, FL, USA, 2010), pp. 1-5

9. IF Akyildiz, D Pompili, T Melodia, State of the Art in Protocol Research for Underwater Acoustic Sensor Networks, 4th edn. (ACM, New York, 2007), pp. 11-12 
10. M Chitre, S Shahabudeen, M Stojanovic, Underwater acoustic communications and networking: recent advances and future challenges. Mar. Technol. Soc. J. 42, 103-116 (2008)

11. N Abramson, The ALOHA system. Proceedings of the AFIPS 1970 Fall Joint Comput Conference 37, 281-285 (1970)

12. L Berkhovskikh, Y Lysanov, Fundamentals of Ocean Acoustics (Springer, New York, 1982)

13. Y Xiao, Y Zhang, JH Gibson, GG Xie, Performance analysis of p-persistent aloha for multi-hop underwater acoustic sensor networks, in International Conference on Embedded Software and Systems, 2009, pp. 305-311

14. Y Xiao, Y Zhang, JH Gibson, GG Xie, H Chen, Performance analysis of ALOHA and p-persistent ALOHA for multi-hop underwater acoustic sensor networks. Cluster Comput. 14, 65-80 (2011)

15. DM Olsson, LS Nelson, The Nelder-Mead simplex procedure for function minimization. Technometrics 17, 45-51 (1975)

16. AF Harris, M Zorzi, Modeling the underwater acoustic channel in ns2, in Proceedings of the 2nd international Conference on Performance Evaluation Methodologies and Tools, vol 321, Nantes, France, 22-27 October 2007 (ValueTools ICST (Institute for Computer Sciences Social-Informatics and Telecommunications Engineering), ICST, Brussels, Belgium, 2007), pp. 1-8

doi:10.1186/1687-1499-2013-65

Cite this article as: Yu et al:: Analyzing the performance of Aloha in string multi-hop underwater acoustic sensor networks. EURASIP Journal on Wireless Communications and Networking 2013 2013:65.

\section{Submit your manuscript to a SpringerOpen ${ }^{\circ}$} journal and benefit from:

- Convenient online submission

- Rigorous peer review

- Immediate publication on acceptance

- Open access: articles freely available online

- High visibility within the field

- Retaining the copyright to your article 